\title{
ZONE OF REACTIONS AS A TOOL IN STUDYING THE SPACE-TIME STRUCTURE OF A FIREBALL
}

\author{
A. Muskeyev* \\ Kyiv Taras Shevchenko National University, 01601 Kiev, Ukraine \\ E-mail: anton.muskeyev@gmail.com \\ D. Anchishkin
}

Bogolyubov Institute for Theoretical Physics, 01601 Kiev,Ukraine

\section{S. Yezhov}

Kyiv Taras Shevchenko National University, 01601 Kiev, Ukraine

\begin{abstract}
We propose an algorithm to determine a zone of hadron reactions during the collision of relativistic nuclei. The zone of reactions is then exploited as a tool in studying the space-time structure of an interacting system (fireball). The relationship of the boundaries of different zones of reactions and the hypersurfaces of sharp chemical and kinetic freeze-outs is discussed. We also consider time-dependence of the reaction rates integrated over spatial coordinates. The evolution of this quantity reveals some interesting features which we explained from a phenomenological point of view. Evaluations are made with the help of the microscopic transport model UrQMD. As an example, we consider the central collisions for AGS (10.8A GeV) and SPS (158A GeV) conditions.
\end{abstract}

XXth Hadron Collider Physics Symposium

November 16 - 20, 2009

Evian, France

* Speaker. 
ZONE OF REACTIONS. We consider a large space-time region $C_{\mathrm{R}}$ which contains a dominant part (99.99\%) of all two-particle reactions and decays of resonances related to the event (a particular nucleus-nucleus collision). In order to determine the zone of reactions, we divide the 4-D cube $C_{\mathrm{R}}$ into separate equal parts (pixels) $\Omega=\Omega(t, \mathbf{x})$, where $(t, \mathbf{x})$ are the coordinates of the center of the pixel. For every space-time point $(t, \mathbf{x})$, one can calculate the absolute number of reactions in the given pixel $\Omega(t, \mathbf{x})$ by using, for instance, a kinetic approach. In the present investigation, we exploit the microscopic transport model UrQMD [1,2]. Hence, for every collision event, we can parametrize the whole amount of pixels with the help of one parameter, the absolute number of reactions which occurred in a pixel. This will be the height of the bin which we put in correspondence to the pixel. Then, we put bins along the $X$-axis in accordance with their parameters: from a pair of bins, the left bin is that, which is higher. Hence, the most tall will be the first on the left. Evidently, the total height of all bins gives us the total number of hadronic reactions $N_{\text {tot }}$ happened in the fireball. Then we map the $(t, \mathbf{x})$ coordinates of pixels (bins) starting from the left of the obtained hierarchy to a 4-volume. We stop moving to the right along the line of bins (pixels) at that point, where the summing up the number of reactions achieves, for instance, $99 \%$ of $N_{\text {tot }}$ (see details in [3]). In such a way, we determine the 4-D region in the space-time, where the most intensive reactions happened. By that, we define the zone of reactions as a part of the 4-cube of reactions $C_{\mathrm{R}}$, in which a certain share (we adopt $99 \%$ ) of all possible processes in the system takes place. It is obvious that the problem of the determination of the reaction region has a statistical character and can be resolved just with some accuracy.

RESULTS AND DISCUSSION. In the present microscopic study with the use of the obtained hierarchy, we separate the following parts of a fireball which characterize its evolution (see Fig. 1): 1) Region of a hot fireball (marked by red color), where $99 \%$ of all inelastic hadronic reactions, $2 \rightarrow 2^{\prime}+m$, have occurred; 2) Region of a cold fireball (blue color) which together with the region of the hot fireball contains $99 \%$ of all hadronic reactions $N_{\text {tot }}$; 3) Additional region of a fireball halo (cyan color), where $0.9 \%$ of all hadronic reactions have occurred. Two last zones together
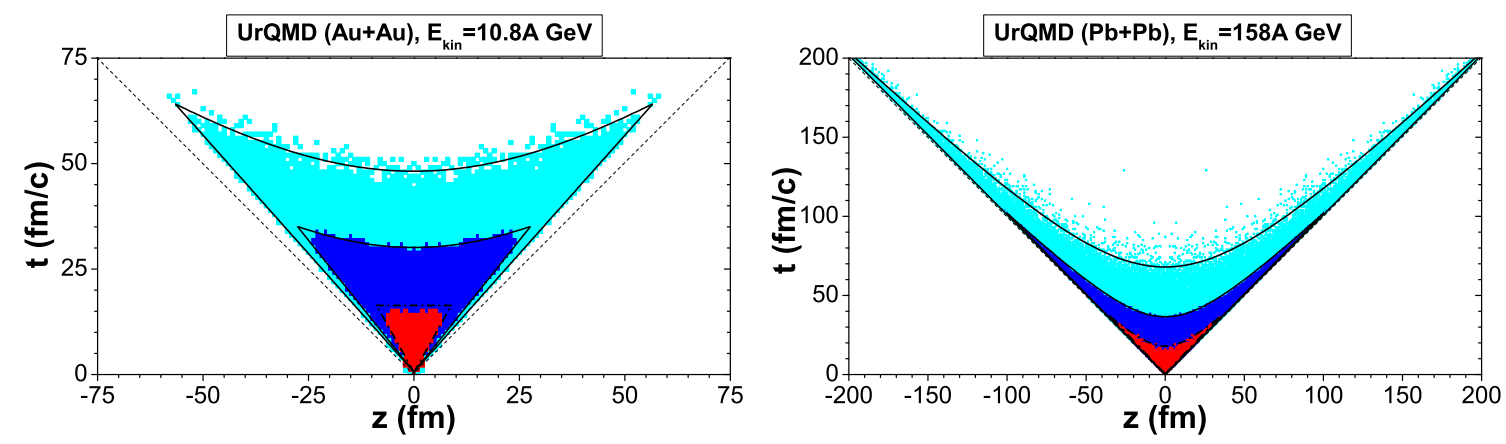

Figure 1: Projections of the zones of reactions on the $z-t$ plane. Red region contains $99 \%$ of all inelastic reactions, $2 \rightarrow 2^{\prime}+m$. Red and Blue regions together contain $99 \%$ of all hadronic reactions. Cyan region contains $0.9 \%$ of all hadron reactions only. Solid lines represent boundaries of the regions. Dashed line bounds the region which contains $90 \%$ of all hadron reactions.

compose a space-time region containing the hadron-resonance gas, and the reactions in this region are mainly presented by decays of resonances. An important question which can be clarified by the 

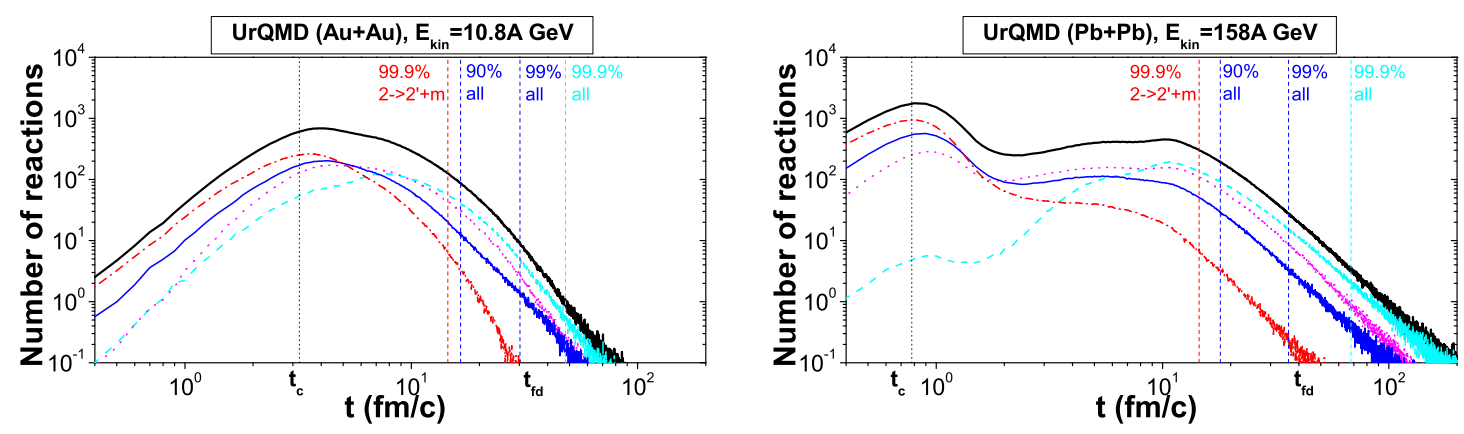

Figure 2: Time dependence of hadron reactions in the fireball for different time moments. The black solid line indicates all reaction rates in the fireball, the blue solid line separates the elastic scattering of hadrons $(2 \rightarrow 2)$, the red line shows all inelastic reactions $\left(2 \rightarrow 2^{\prime}+m\right.$, where $\left.m \geq 0\right)$, the magenta line stands for fusion reactions $\left(2 \rightarrow 1^{\prime}\right)$, and the cyan distinguishes decays $\left(1 \rightarrow 2^{\prime}+m, m \geq 0\right)$. Vertical dashed lines are used to depict boundaries of the zones of reactions at $z=0$.

study of the zone of reactions is how the space-time boundary of a fireball is related to the so-called sharp freeze-out hypersurface. The sharp kinetic freeze-out hypersurface is usually defined with the help of some parameter $P(t, \mathbf{x})$ which takes the critical value $P_{c}$ on the hypersurface. Besides, one can follow the "classical" definition: the sharp kinetic freeze-out hypersurface is an imaginary hypersurface, outside of which there are no collisions between particles of the system. Obviously, this hypersurface can be determined with some accuracy. If we take this accuracy as $99 \%$ of the total number of hadron reactions which take place during a nucleus-nucleus collision, then we can identify two boundaries of the zone of reactions and the sharp freeze-out hypersurfaces, chemical and kinetic ones. Hence, in the framework of the above definition, the chemical freezeout hypersurface is the boundary between the hot (red) and cold (blue) fireball zones. In the same way, the boundary between the cold (blue) fireball zone and the fireball halo (cyan color) can be put in correspondence to the sharp kinetic freeze-out hypersurface. The space-time part of the kinetic hypersurface is a hyperbola and has form $t(z)=A \sqrt{\tau_{0}^{2}+z^{2}}$, where $A=0.75, \tau_{0}=64 \mathrm{fm} / \mathrm{c}$ for the AGS-energy $E_{\text {kin }}=10.8 \mathrm{~A} \mathrm{GeV}$ and $A=0.95, \tau_{0}=38 \mathrm{fm} / \mathrm{c}$ for the SPS-energy $E_{\text {kin }}=158 \mathrm{~A} \mathrm{GeV}$.

Of significant interest is the evolution of the different reaction rates integrated with respect to the spatial coordinates, see Fig. 2. A specific feature of the total reaction integral rate (thick solid line) is its initial increase. We explain this by the increase of the number of nucleons as participants of the reactions, when one nucleus penetrates into another one. The maximum happens at the time moment $t=t_{c} \equiv R_{0} / \gamma v$, where $R_{0}$ is the nucleus radius, $v=p_{z} /\left(m_{N}^{2}+p_{z}^{2}\right)^{1 / 2}$, $\gamma=\left(1-v^{2}\right)^{-1 / 2}, p_{z}$ is the initial nucleon momentum in the c.m.s. of two nuclei, and $m_{N}$ is the nucleon mass. We name $t_{c}$ as the fireball formation time. For experiments under consideration, this gives the time moments $t_{c}=3.2 \mathrm{fm} / \mathrm{c}$ for AGS $(10.8 \mathrm{~A} \mathrm{GeV})$ and $t_{c}=0.78 \mathrm{fm} / \mathrm{c}$ for SPS $(158 \mathrm{~A} \mathrm{GeV})$ which practically coincide with the times of the first maximum, see Fig. 2.

\section{References}

[1] S.A. Bass, M. Belkacem, M. Bleicher et al., Prog. Part. Nucl. Phys. 41 (225) 1998.

[2] M. Bleicher, E. Zabrodin, C. Spieles et al., J. Phys. G: Nucl. Part. Phys. 25 (1859) 1999.

[3] D.V. Anchishkin, A.O. Muskeyev, S.N. Yezhov, arXiv:0908.1723 [nucl-th]. 